\title{
In vitro effects of vasoconstrictive retraction agents on primary human gingival fibroblasts
}

\author{
DANUTA NOWAKOWSKA ${ }^{1}$, JOLANTA SACZKO ${ }^{2}$, ANNA SZEWCZYK ${ }^{2,3}$, OLGA MICHEL ${ }^{4}$, \\ MAREK ZIĘTEK $^{5}$, JOANNA WEŻGOWIEC ${ }^{5}$, WŁODZIMIERZ WIĘCKIEWICZ ${ }^{1}$ and JULITA KULBACKA ${ }^{2}$ \\ ${ }^{1}$ Department of Prosthodontics, Wroclaw Medical University, Wroclaw 50-425; ${ }^{2}$ Department of Molecular and \\ Cellular Biology, Wroclaw Medical University, Wroclaw 50-556; ${ }^{3}$ Department of Animal Developmental Biology, \\ Institute of Experimental Biology, University of Wroclaw, Wroclaw 50-335; ${ }^{4}$ Department of \\ Medical Biochemistry, Wroclaw Medical University, Wroclaw 50-368; ${ }^{5}$ Department of \\ Experimental Dentistry, Wroclaw Medical University, Wroclaw 50-425, Poland
}

Received March 27, 2019; Accepted September 17, 2019

DOI: $10.3892 /$ etm.2020.8462

\begin{abstract}
The biological activity of chemical retraction/ displacement agents in surrounding periodontal tissues is of unquestionable importance, but the activity of these agents has not been completely elucidated. In the present study, we aimed to evaluate the in vitro effects of vasoconstrictive retraction agents on primary human gingival fibroblasts (HGFs). A total of six commercial adrenergic solutions $(0.05$ and $0.01 \%$ $\mathrm{HCl}$-epinephrine, two based on $0.05 \% \mathrm{HCl}$-tetrahydrozoline, $0.05 \% \mathrm{HCl}$-oxymetazoline, and $10 \% \mathrm{HCl}$-phenylephrine) and three experimental gel formulations (EG-1, EG-2, and EG-3) were used to treat primary HGFs. The biological effect of the retraction treatment on the expression of collagen types I and III was detected by performing immunocytochemical analysis. The generation of reactive oxygen species triggered by the retraction agents were evaluated by using the dichlorofluorescein (DCF) fluorescent probe. The effect of retraction agents on the expression of fibronectin was visualized by confocal laser scanning microscopy. According to the results, experimental retraction gels did not limit the expression of collagen types I and III. EG-3 even induced the synthesis of both types of collagen. The DCF assay indicated oxidative stress similar to the control cells for most of the selected retraction agents. Experimental gels did not cause degradation of the cellular shape and morphology of the primary HGFs. The proposed experimental retraction gels in the present study demonstrated higher biocompatibility with primary HGFs, suggesting their use as clinical vasoconstrictive agents for the application of gingival retraction with minimal damage to periodontal tissues.
\end{abstract}

Correspondence to: Professor Jolanta Saczko, Department of Molecular and Cellular Biology, Wroclaw Medical University, ul. Borowska 211A, Wroclaw 50-556, Poland

E-mail: jolanta.saczko@umed.wroc.pl

Key words: gingival margin retraction, human gingival fibroblasts, collagen types I and III, oxidative stress, fibronectin

\section{Introduction}

Temporary changes in the architecture of the free gingival margin, as well as the gingival crevicular fluid flow and the control of bleeding effect into gingival sulcus, are necessary for the precise treatment procedures in restorative dentistry. Past several decades have witnessed the use of chemo-mechanical methods with various retraction/displacement media and chemical retraction/displacement agents by dentists (1). This technique provides optimal conditions for imaging and transmission of morphological status of the prepared tooth and/or design of the implant's structure and the surrounding periodontal configuration to the dental laboratory through impression materials or optical/digital scanning in the patient's mouth in the computer-aided design/computer-aided manufacturing (CAD/CAM) techniques (2).

In dental practice, two categories of chemical retraction agents are used: Conventional retraction agents (CRAs) (e.g., astringents (e.g., coagulants and hemostatics)) and experimental retraction agents (ERAs) (e.g., vasoconstrictors (e.g., adrenergics)) $(3,4)$. The astringents contain inorganic metallic salts such as aluminum chloride and sulfate, ferric sulfate, and others. From the clinical point of view, CRAs are very effective agents. However, they have been shown to have numerous adverse-reversible and irreversible-local effects on the gingival tissue, which are associated with the low acidity of the astringents $(\mathrm{pH}<3)(5-10)$. The vasoconstrictors used previously were based on the organic salts of $\mathrm{HCl}$, as well as on the $\alpha$ - and $\beta$-adrenergics (HCl-epinephrine) and $\alpha$-adrenergics (HCl-tetrahydrozoline,-oxymetazoline, and-phenylephrine). Recently, $\mathrm{HCl}$-xylometazoline was also experimentally verified $(11,12)$. The most popular $\mathrm{HCl}$-epinephrine has been used at different concentrations. Few studies have reported that $4 \%$ epinephrine can induce systemic side effects manifested as 'Epinephrine Syndrome' $(13,14)$. Fazekas et al (15) studied $0.1 \% \mathrm{HCl}$-epinephrine and Csillag et al (16) studied $0.01 \% \mathrm{HCl}$-epinephrine and reported that these agents showed vasoconstrictor response in gingival tissues with reduced systemic side effects. Moreover, it has been shown that the exo-and endogenic effects of epinephrine can be accumulated 
in human body during gingival margin retraction procedures. Bowles et al (17) proposed $\alpha$-adrenergic sympathomimetic amines as potential alternative retraction agents with effective constriction of gingival blood vessels and minimal systemic action.

Previous studies on the comparative histological evaluation of the response of gingival tissue in beagle dogs and rabbits after exposure to the selected CRAs and ERAs revealed a low damaging potential of $0.05 \% \mathrm{HCl}$-tetrahydrozoline (18-20). Several in vitro studies on various cell lines have shown a significantly higher cytotoxicity of astringents than that of the vasoconstrictors (21-26). A previous study compared the cytotoxic effects on primary human gingival fibroblasts (HGFs) by using selected chemical retraction agents and demonstrated the following array: $0.01 \% \mathrm{HCl}$-epinephrine $<0.1 \% \mathrm{HCl}$-epinephrine $<5 \%$ aluminum sulfate $<20 \%$ aluminum sulfate $<15.5 \%$ ferric sulfate (24). All these aforementioned studies evaluated the cytotoxicity of chemical retraction agents only in the solution form. However, Nowakowska et al investigated the dynamic response of primary HGFs after treatment with CRAs and ERAs in solution and gel formulations $(25,26)$. They isolated HGFs from healthy gingival tissues by the method described by Saczko et al (27) and incubated the cells with retraction agents for 3, 5, and $10 \mathrm{~min}$, according to the clinical habits of dentists performing gingival retraction, as well as for $24 \mathrm{~h}$. MTT ((3-(4,5-dimethylthiazol-2-yl)-2,5-diphenyltetrazolium bromide) tetrazolium reduction)) assay was performed to test the cytotoxicity $(27,28)$. After $10 \mathrm{~min}$ of exposure to both the evaluated groups of chemicals, the mitochondria of HGFs showed a higher activity, which suggests an increase in their antioxidative defense capabilities. In a subsequent in vitro study, it was demonstrated that the cytotoxicity of the evaluated vasoconstrictor retraction agents decreased in the following order: $0.1 \% \mathrm{HCl}$-epinephrine >parallel 0.01 and $0.05 \%$ $\mathrm{HCl}$-epinephrine $>\alpha$-sympathomimetic amine solutions $>0.05 \%$ $\mathrm{HCl}$-tetrahydrozoline gels. The minimal cytotoxic effect on the mitochondrial oxidoreductive potential was demonstrated by three self-prepared experimental gels at all evaluated time periods, including $24 \mathrm{~h}$, and the differences in the cell viability after treatment with gels were not statistically significant (26).

The biological activity of the chemical retraction agents in the surrounding periodontal tissues is a crucial factor. However, their mechanism of action has not been completely clarified yet. The results presented in the previous studies based on in vitro experiments have expanded the knowledge on the action of vasoconstrictive retraction agents in primary HGFs $(1,26,29)$. Through the assessment of selected oxidative stress markers, such as lipid peroxidation (the concentration of malondialdehyde (MDA)), protein damage (-SH), colony formation, and the expression of manganese superoxide dismutase (MnSOD), it was concluded that the experimental gels induced oxidative changes in primary HGFs at the lowest level. The evaluation of proteins (F-actin and $\beta$-tubulin) in the cellular cytoskeleton of primary HGFs after $24 \mathrm{~h}$ of incubation with gingival retraction agents showed that the studied vasoconstrictive chemical retraction agents can have a cytotoxic potential toward gingival tissue under clinical condition. These observations of the rearrangement of the cytoskeleton also suggested that the experimental gels caused degradation of the cellular structure of primary HGFs (29).
Therefore, in the present study, we aimed to evaluate, in more detail, if the proposed vasoconstrictive experimental gels are more biocompatible with the gingival margin tissue and if they can be applied as minimally invasive chemical retraction agents. In addition, we propose an experimental approach that can be used as a validation method in the evaluation of biocompatibility of the newly developed retraction agents. HGFs were established as a research model for this study to enable a comparison of the results with the other studies concerning issue of biocompatibility of gingival retraction agents. Observation of collagen and fibronectin expressed by HGFs was performed in order to evaluate the influence of the selected retraction agents on the organization of cytoskeleton and the extracellular matrix. Additionally, the level of reactive oxygen species (ROS) was determined using the DCF assay.

\section{Materials and methods}

Chemical retraction agents. Six adrenergic commercial solutions and three self-prepared experimental gel formulations-EG-1, EG-2, and EG-3 (patent No P. 397505 'Dental composition')-were studied. Table I shows their characteristics. These chemicals were diluted with Dulbecco's modified Eagle's medium (DMEM) to 1:20 ratio, which was further studied based on our previous studies $(1,25)$.

Cell culture. Primary HGFs were mechanically isolated from a fragment of gingival tissue (1-2 $\mathrm{mm}$ ) obtained from healthy patients, according to the procedure described by Dominiak and Saczko (27). The biopsies were provided by the Department of Dental Surgery of the Faculty of Medicine and Dentistry of Wroclaw Medical University in accordance with the requirements of the Bioethics Commitee of the Wroclaw Medical University. Patients were two women and one men, who were subjected to surgery for tooth extraction and agreed as volunteers for tissue biopsy (Bioethical Committee approval, no: KB-8/2010). Patients were recruited from January till December 2018, they were healthy people in the age between 18-50. All patients provided their informed consent to participate in the present study. No differences were observed in experimental protocol depending on the patients' sex. Inclusion criterion was healthy periodontium of the tissue donors, and exclusion criterion were diseases of gingival tissues and/or oral cavity tissues. HGFs from several different donors were used for different repetitions of experiments, however, the results were not distinguished on the basis of the cells donor. Before surgery, gingival tissue was rinsed by boric acid, to avoid mycological infection. The taken biopsy did not exceed $3 \times 2 \times 2 \mathrm{~mm}$. The tissue was taken by a scalpel and immediately placed in a cell culture medium (Dulbecco's modified Eagle's medium, DMEM) containing 10\% fetal bovine serum (FBS), and antibiotics penicillin/streptomycin (Sigma). Primary cells were grown on Petri dishes $(60 \mathrm{~mm}$, Nunc) and during the next passages routinely in $25 \mathrm{~cm}^{2}$ flasks (Equimed). The cells were maintained in a humidified atmosphere at $37^{\circ} \mathrm{C}$ and $5 \% \mathrm{CO}_{2}$. For experimental reasons, the cells were detached by trypsinization ( $0.25 \%$ trypsin-EDTA, Sigma). Successfully establishing of HGFs was confirmed in immunofluorescent images of cells stained for fibronectin, one of the markers of HGFs. The obtained primary cell cultures 
were frozen in $-80^{\circ} \mathrm{C}$ (up to 3 months) or in $-160^{\circ} \mathrm{C}$ (up to 6 months) using standard protocol with Bambanker freezing medium (ABO).

Immunocytochemical evaluation of collagen types I and III. The biological effect of the selected retraction agents was determined by the evaluation of the expression of collagen types I and III by immunocytochemical (ABC) method using DAKO kit (LSAB®2 System-HRP, cat. no. K0673) based on the labelled streptavidin-biotin (LSAB) method in which a biotinylated secondary antibody reacts with several peroxidase-conjugated streptavidin molecules. Primary HGFs (1,000 cells) were seeded onto 8-well slides (Equimed) and then incubated with retraction agents for $24 \mathrm{~h}$ in $37^{\circ} \mathrm{C}$. Next, the fibroblasts were fixed using $4 \%$ paraformaldehyde (PFA, Roth) for $10 \mathrm{~min}$ at room temperature. Collagen types I and III were visualized with the polyclonal goat antibodies (Collagen types I (cat. no. sc-59772) and III (cat. no. sc-271249); Santa Cruz Biotechnology). The incubation with primary antibodies was performed overnight in $4^{\circ} \mathrm{C}$. After incubation the staining protocol was applied according to the manufacturer (DAKO) requirements in RT. DAB (3,3-diaminobenzidine) was utilized for immunodetection ( 5 min of incubation in RT) and hematoxylin (Roth) for nuclei staining (1 min of incubation in RT). At the end the dehydration (5 min in each of 6 various concentrations of ethanol) and transparentizing steps (3x5 min) were performed in RT and finally microscopic slides were mounted with DPX medium (Sigma-Aldrich) and were examined using an upright microscope Olympus BX51 using $20 \mathrm{x}$ or $40 \mathrm{x}$ magnification. The percentages of the stained cells were obtained by counting 100-200 cells in three randomly selected fields. Each slide was examined by two independent investigators. Cells were evaluated as positive if the stained reaction was noted in more than 5\% of the cells (30-32). The intensity of immunocytochemical staining was rated as follows: (-) negative (no stained reaction), $(+)$ weak, $(++)$ moderate, $(++/+++)$ higher than moderate, and $(+++)$ strong. Negative controls with phosphate buffered saline (PBS, Lab Empire, Poland) were prepared on each slide.

Dichlorofluorescein (DCF) assay. We determined the level of reactive oxygen species (ROS) using the DCF assay (Life Technologies), which was conducted with 6-carboxy-2,7-dichlorodihydrofluorescein diacetate (carboxy-H2DCFDA). Briefly, the stock solution of carboxy-H2DCFDA $(50 \mu \mathrm{g} / \mathrm{ml}$ in sterile DMSO (Sigma)) was prepared at room temperature in the dark and then diluted using the cell culture medium without FBS. Cells were cultivated on black 96-well plates with transparent bottom overnight before the experiment to achieve $70 \%$ of confluency. For experimental protocol retraction agents were diluted in cell culture medium (DMEM) to 1:20 ratio. Then cells were incubated with gingival retraction agents and reactive oxygen species were measured after 5, 20, and $25 \mathrm{~min}$ of exposition. After washing out of the incubation medium from cells using PBS with $6 \mathrm{mM}$ glucose, the working solution of carboxy-H2DCFDA was added to the cell culture medium to a final concentration of $10 \mu \mathrm{M}$ and the cells were incubated at $37^{\circ} \mathrm{C}$ in darkness for $30 \mathrm{~min}$. After this, the excitation and emission were measured at 495 and $530 \mathrm{~nm}$, respectively. ROS level was detected after 5, 20, and $25 \mathrm{~min}$ 


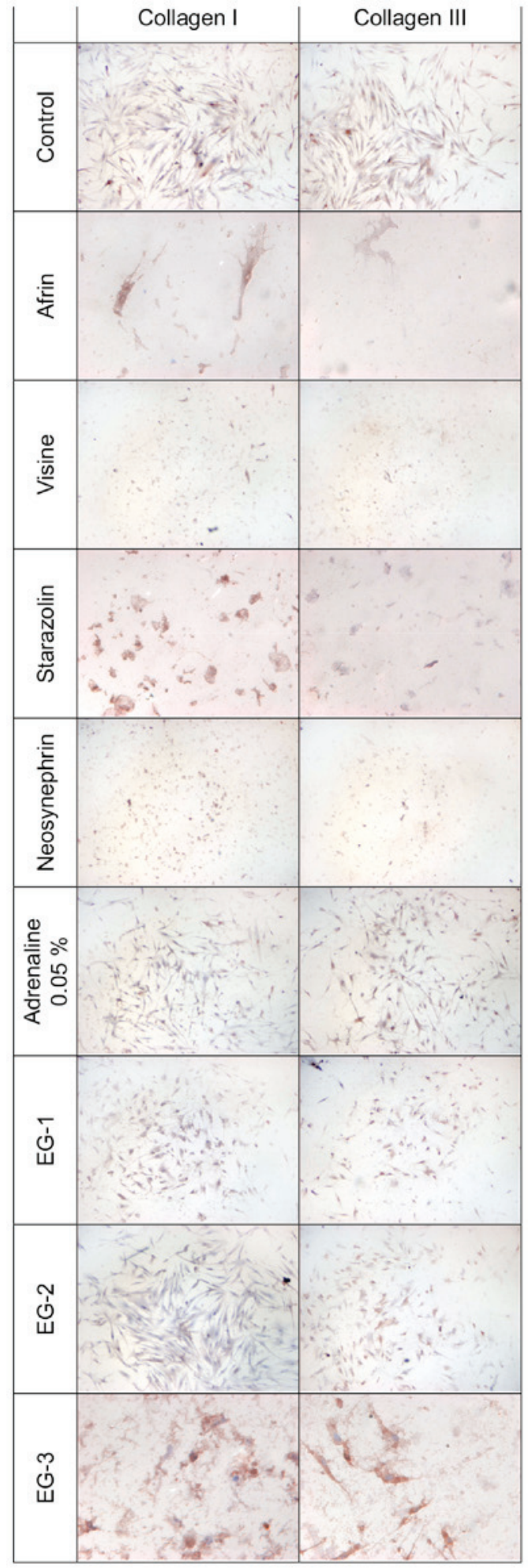

Figure 1. Evaluation of the expression of collagen I and III in human gingival fibroblasts after $24 \mathrm{~h}$ of incubation with gingival retraction agents diluted with the cell culture medium (DMEM) to a 1:20 concentration. A microscope was used for the visualization of the Control, Visine, Neosynephrin, Adrenaline $0.05 \%$, EG-1 and EG-2 groups. Magnification, x20. A microscope was used of the visualization for the Afrin, Starazolin and EG-3 groups. Magnification, x40. EG, experimental gel.

of treatment with retraction agents by a multiwell scanning spectrophotometer (EnSpire Perkin Elmer). All results were compared to the control untreated cells cultivated in the same conditions. The results were expressed as a mean values of measured fluorescence intensity.

Confocal laser scanning microscopy for the evaluation of fibronectin. For the evaluation of distribution of fibronectin, the following procedure of immunofluorescence was performed. Primary HGFs (1,000 cells) were grown on coverslips for $24 \mathrm{~h}$ at $37^{\circ} \mathrm{C}$, and then incubated with retraction agents diluted in the culture medium for $24 \mathrm{~h}$ at $37^{\circ} \mathrm{C}$. Next, the fibroblasts were washed with PBS (5 min at room temperature), fixed using 4\% PFA in PBS (10 min at room temperature), blocked with $1 \% \mathrm{FBS}$ in PBS (for $1 \mathrm{~h}$ at $37^{\circ} \mathrm{C}$ ), and permeabilized with $1 \%$ triton X-100 (Sigma) in PBS (v/v) (3x3 min at room temperature). All washing steps were performed with PBS. After an overnight incubation of cells with mouse monoclonal anti-fibronectin antibody [IST-9] (ab6328, diluted 1:200; Abcam) at $4^{\circ} \mathrm{C}$, the cells were washed with PBS (2x10 min at room temperature) and labeled with antibody Alexa Fluor ${ }^{\circledR}$ 488 AffiniPure Goat Anti-Mouse IgG (H+L) (115-545-003, diluted 1:100, Jackson ImmunoResearch) for $1 \mathrm{~h}$ at $37^{\circ} \mathrm{C}$. Then, the cells were mounted in a fluorescence mounting medium with 4',6-diamidino-2-phenylindole dihydrochloride (DAPI, Sigma) for DNA staining. For the imaging of the fibronectin distribution, Olympus FluoView FV1000 confocal laser scanning microscope (Olympus) with 60x magnification was used.

Statistical analysis. The data are presented as mean \pm error. The minimum number of repeats performed was $n=9$. The evaluation of statistical significance was performed by two-way ANOVA with Tukey post-hoc test using the control group represented by the untreated HGFs-cells incubated with a culture medium (DMEM) without any retraction agent. The evaluation involved separate controls for each time point in DCF assay. $\mathrm{P}<0.05$ was considered to indicate a statistically significant difference. GraphPad Prism 7.0 software was implemented for the analysis.

\section{Results}

Expression of collagen types I and III. Fig. 1 presents the results of experimental collagen types I and III evaluation in primary HGFs. Table II presents semi-quantitative results. The results of the expression of collagen types I and III in primary HGFs were determined after $24 \mathrm{~h}$ of incubation with gingival retraction agents using 1:20 dilution. A slight increase in collagen types I and III quantity was observed in HGFs after $24 \mathrm{~h}$ of incubation with all the investigated retraction agents. The highest intensity of immunocytochemical reaction was observed after incubation with the experimental gels (EG-1, EG-2, and EG-3).

Level of ROS. The effect of retraction agents on the generation of free radicals and promotion of oxidative stress was examined. Oxidative stress was evaluated by DCF assay after different times of incubation $(5,20$, and $25 \mathrm{~min})$ with various retraction agents using 1:20 concentration. The increase in the level of free radicals was proportional to the time of incubation in HGFs. In most cases, the level of increase of ROS after incubation with retraction agents did not differ from that of the control cells (Fig. 2). The newly developed experimental gels 
Table II. Evaluation of the expression of collagen types I and III in primary human gingival fibroblasts after $24 \mathrm{~h}$ of incubation with gingival retraction agents diluted with the cell culture medium (DMEM) to 1:20 concentration.

\begin{tabular}{|c|c|c|c|c|}
\hline \multirow[b]{2}{*}{ Gingival retraction agent } & \multicolumn{2}{|c|}{ Collagen type I } & \multicolumn{2}{|c|}{ Collagen type III } \\
\hline & Positive cells, $\%$ & Reaction intensity & Positive cells, $\%$ & Reaction intensity \\
\hline Control & 98 & $++/+++$ & 97 & +++ \\
\hline Afrin & 100 & ++ & 100 & + \\
\hline Visine & 100 & + & 100 & + \\
\hline Starazolin & 100 & + & 100 & $-/+$ \\
\hline Neosynephrin & 100 & ++ & 100 & + \\
\hline Adrenaline $0.05 \%$ & 100 & + & 100 & + \\
\hline EG-1 & 100 & $++/+++$ & 100 & +++ \\
\hline EG-2 & 100 & ++ & 100 & $++/+++$ \\
\hline EG-3 & 100 & ++ & 100 & $++/+++$ \\
\hline
\end{tabular}

The intensity of immunocytochemical staining was rated as follows: i) (-) Negative (no stained reaction), ii) (+) weak; iii) (++) moderate; iv) $(++/+++)$ higher than moderate; and v) $(+++)$ strong. EG, experimental gel.

stimulated the production of ROS similar to the commercial retraction agents (e.g., Neosynephrin or Visine).

Evaluation of fibronectin distribution. We performed a semi-quantitative determination of fibronectin as a marker of cell attachment and proliferation.

Fig. 3 presents the results of evaluation of the intracellular distribution of fibronectin in HGFs after $24 \mathrm{~h}$ of incubation with selected gingival retraction preparations using immunofluorescence technique. The results show that Neosynephrin was the most cytotoxic agent, which caused disorders of the cellular morphology and significant antiproliferative effect. Incubation of HGFs with Visine slightly disturbed the expression of fibronectin. Other studied retraction agents had no significant influence on the distribution of fibronectin. However, we were able to observe that EG-2 stimulated the expression of fibronectin. Our results confirm the safety of using the studied experimental gels. Moreover, our results suggest the beneficial effect of EG-2.

\section{Discussion}

Vasoconstrictive gingival retraction agents should have a sufficient clinical efficiency without local and minimal systemic side effects (18-20). A systemic reaction is infrequent in some vasoconstrictor materials of the alpha agonists-including tetrahydrozoline, oxymetazoline, and phenylephrine-that are commonly used as eye and nose decongestant drops. Therefore, a dose that is lower than the maximum allowed can be used in the gingival retraction. Bowles et al (17) reported that tetrahydrozoline is a powerful retraction agent. Similar investigations have shown that tetrahydrozoline is better than epinephrine for gingival retraction protocols $(15,16)$. However, in clinical practice, the vasoconstrictive gingival retraction agents are available only in solution form.

Previously, we demonstrated that cytotoxic effect of the product is dependent on the purity of the active substance and the pharmacological form of chemical agents (26). In this study, we focused on the biological properties of the experimental gels EG-1, EG-2, and EG-3 in comparison with the commonly used vasoconstrictive retraction agents-epinephrine and experimental $\alpha$-adrenergic sympathomimetic amines solutions. The experimental gels are based on the pure form of the active substance-tetrahydrozoline, contrary to other retraction agents used in prosthodontics, such as Visine classic and Starazolin. All these compounds belong to sympathomimetic amines, which contain other chemical substances in addition to tetrahydrozoline. We hypothesized that these chemical differences can influence and modify the biological effects of retraction agents in gingival fibroblasts, which have been shown in our previous studies $(26,29)$. In our previous in vitro study, the examination revealed minor cytotoxic effect of the experimental gels in contrast to the commercial preparations (Visine, Starazolin, Afrin, and Neosynephrin) (26). Based on these results, three gels containing $0.05 \% \mathrm{HCl}$-tetrahydrozoline were prepared. In this study, we performed additional experiments to evaluate the cytotoxicity and biocompatibility of our experimental gels in more detail. To investigate whether cells exposed to retraction agents show increased level of oxidative stress, we measured the level of ROS with DCF assay. In most cases, the level of ROS after incubation with retraction agents was on similar to the control cells. Several in vitro tests can be used to determine the cytotoxicity of the biomaterial. Regarding oral tissues and practical motives, cell culture model seems to be more appropriate than that of in vivo studies as it enables the performance of multiple tests for the estimation of precise biological response (26).

Furthermore, in this study, we focused on the evaluation of the influence of the selected retraction agents on the organization of cytoskeleton and the extracellular matrix. Cytoskeleton proteins play a very important role in the proper functioning of cells as these proteins provide the structural framework for the cell and are responsible for the movement of cell and internal transport. In this study, we aimed to evaluate the expression of fibronectin and two types of collagen (types I and III). Our evaluation showed that some of the studied retraction agents 


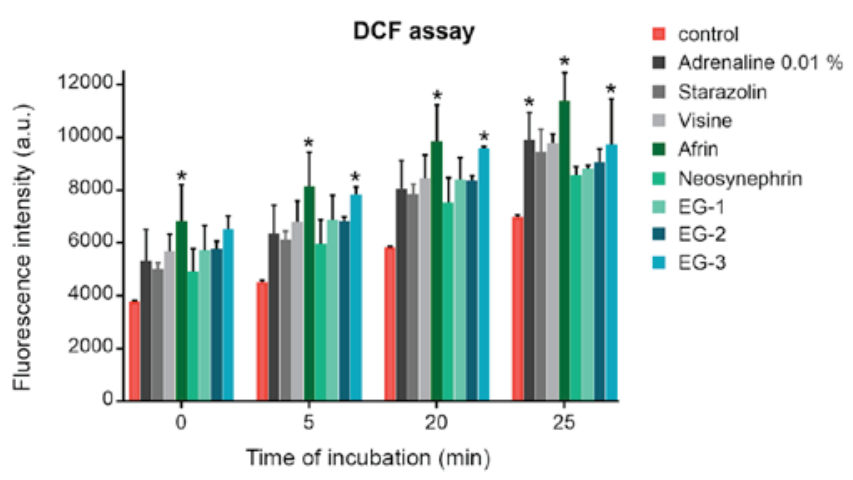

Figure 2. Level of reactive oxygen species detected by DFC assay in human gingival fibroblasts after various time points of incubation with gingival retraction agents in a 1:20 ratio. DCF, dichlorofluorescein; EG, experimental gel.

(Visine and Neosynephrin) influenced the expression of fibronectin, which can lead to disorders in the construction of the cytoskeleton. However, none of the experimental preparations (EG-1, EG-2, and EG-3) altered the intracellular arrangement and fluorescent signal of fibronectin. We observed numerous cells with normal cytoplasm and regular distribution of fibronectin after incubation with new experimental gels. We also observed that EG-2 stimulated the expression of fibronectin. These results confirm that the studied experimental gels are biologically safe. Moreover, our results suggest a beneficial effect of EG-2 on cellular matrix proteins. Fibronectin, binds with the components of the extracellular matrix such as collagen and fibrin and is very important for wound healing. It also plays a key role in cell adhesion, growth, and migration (33). The results of this study demonstrated a slight increase in the amount of collagen types I and III in HGFs after $24 \mathrm{~h}$ of incubation with the newly investigated retraction agents (EG-1, EG-2, and EG-3). This indicates that tetrahydrozoline contained in the gel formulation plays a double role: it not only acts as the retraction agent but also plays a role in wound healing by increasing the expression of collagen and fibronectin.

Our study revealed that the oxidative changes induced by the experimental gel formulations were on the physiological level. Moreover, the observations of the cytoskeleton of HGFs clearly indicated that the experimental gels did not affect their cellular structure and they even stimulated the expression of collagen and fibronectin.

For the proper understanding of the mechanism of action of retraction agents, it is necessary to recognize the limitations of in vivo and in vitro study. In clinical conditions, sulcular epithelium and subepithelial connective tissue protect the gingival fibroblasts. In addition, the concentration of the chemical retraction agents might get decreased due to the constant humidity and temperature maintained in the oral cavity. Moreover, the applied agents can get washed away by the crevicular fluid flow, due to bleeding, or via saliva. Probably, the systemic blood circulation parameters can also affect the successful application of the retraction procedure. Therefore, the same vasoconstrictor retraction agents may act in an unusual way under clinical conditions. Further clinical studies are required to extend the results presented in this article and to find their clinical relevance.
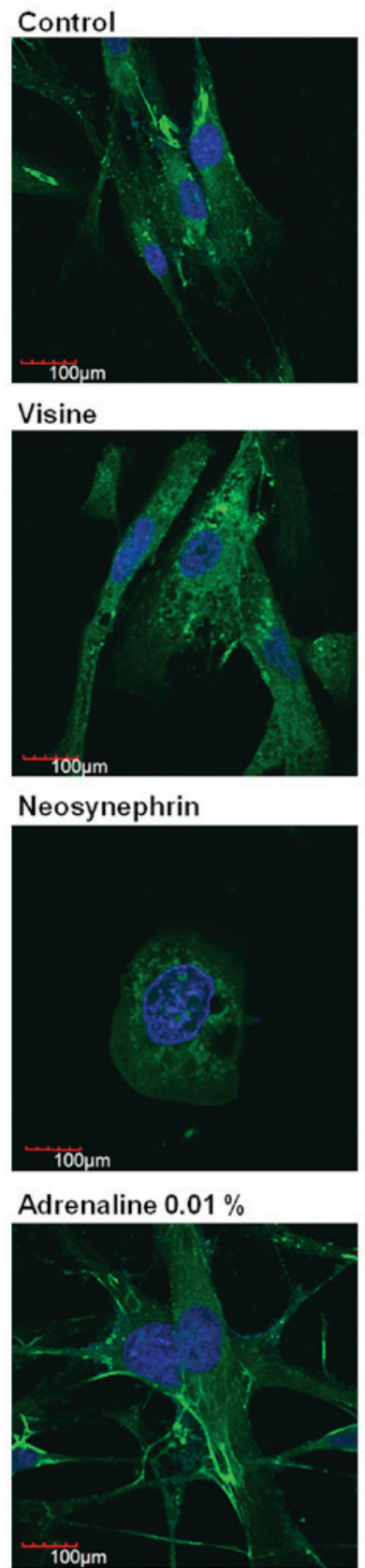

EG-2
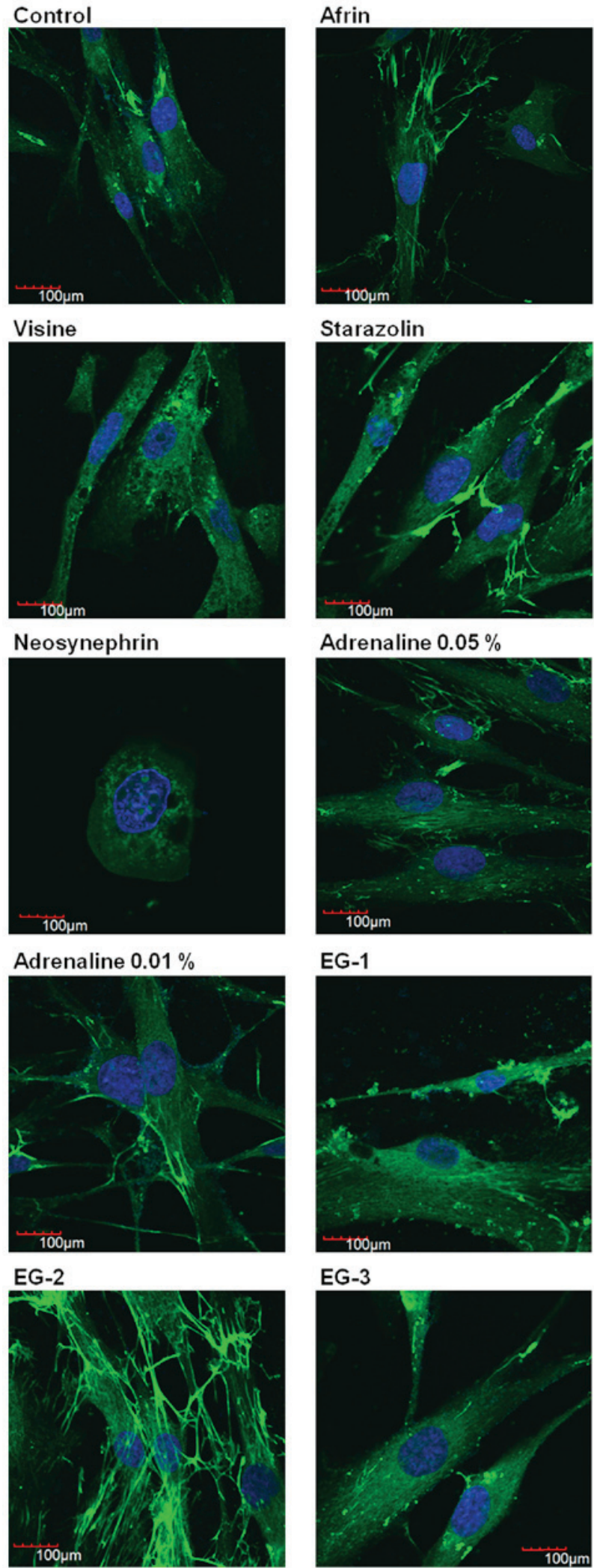

Adrenaline $0.05 \%$

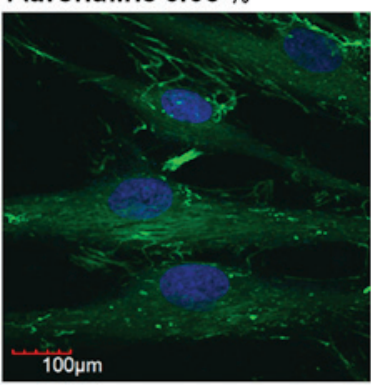

EG-1

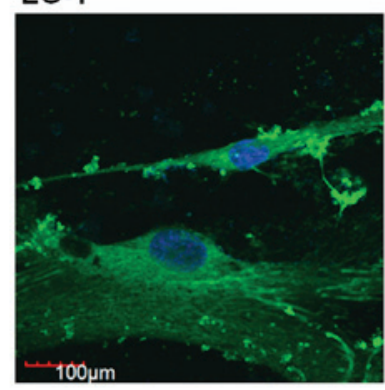

EG-3

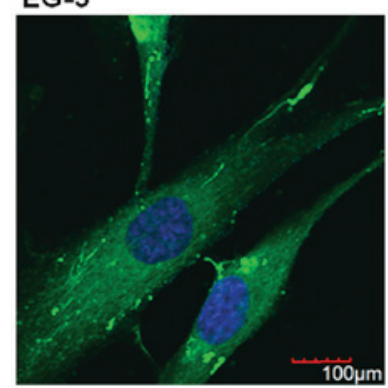

Figure 3. Evaluation of fibronectin distribution by confocal microscopy in human gingival fibroblasts after $24 \mathrm{~h}$ of incubation with gingival retraction agents diluted in the cell culture medium (DMEM) to 1:20 concentration. Green indicates fibronectin and blue indicates DAPI. EG, experimental gel.

To conclude, regardless of the limitations of this in vitro study, the results suggest that the proposed experimental gels are biocompatible with periodontal tissues, and they can be 
considered as the new vasoconstrictive chemical retraction agents.

\section{Acknowledgements}

Not applicable.

\section{Funding}

The present study was supported by Funds of Department of Molecular and Cellular Biology, Wroclaw Medical University (grant no. 846-183) and Funds of Department of Prosthodontics, Wroclaw Medical University (grant no. ST-365).

\section{Availability of data and materials}

All data generated or analyzed during the present study are included in this published article.

\section{Authors' contributions}

DN, JS and JK contributed to the conception and design of the study, analysis and interpretation of data, drafting the article and final approval of the submitted version. AS and $\mathrm{OM}$ contributed to the acquisition of data, revising the article critically for important intellectual content and final approval of the submitted version. MZ and WW contributed to the conception and design of the study, revising the article critically for important intellectual content and final approval of the submitted version. JW contributed to the acquisition, analysis and interpretation of data, drafting the article, and final approval of the submitted version.

\section{Ethics approval and consent to participate}

All healthy donor patients provided their informed consent to participate in this study in accordance with The Declaration of Helsinki. The present study was planned with no risk and in agreement with the Wroclaw Medical University Bioethical Committee. All patients signed agreement before the examination. All patients provided their informed consent to participate in the present study. Wroclaw Medical University Bioethical Committee approved the present study (approval no. KB-8/2010).

\section{Patient consent for publication}

Not applicable.

\section{Competing interests}

The authors declare that they have no competing interests.

\section{References}

1. Nowakowska D, Saczko J, Kulbacka J and Więckiewicz W: Chemical Retraction Agents-in vivo and in vitro studies into their physico-chemical properties, biocompatibility with gingival margin tissues and compatibility with elastomer impression materials. Mini Rev Med Chem 17: 435-444, 2017.

2. Bennani V, Schwass D and Chandler N: Gingival retraction techniques for implants versus teeth: Current status. J Am Dent Assoc 139: 1354-1363, 2008.
3. Felpel LP: A review of pharmacotherapeutics for prosthetic dentistry: Part I. J Prosthet Dent 77: 293-305, 1997.

4. Nowakowska D: Classification of chemical retraction agents. Protet Stomatol 58: 202-208, 2008 (In Polish).

5. Phatale S, Marawar PP, Byakod G, Lagdive SB and Kalburge JV: Effect of retraction materials on gingival health: A histopathological study. J Indian Soc Periodontol 14: 35-39, 2010.

6. Chaudari J, Prajapati P, Patel J, Sethuraman R and Naveen YG: Comparative evaluation of the amunt of gingival displacement produced by three different retraction systems: An in vivo study. Contemp Clin Dent 6: 189-195, 2015.

7. Akca EA, Yildirim E, Dalkiz M, Yavuzyilmaz H and Beydemir B: Effects of different retraction medicaments on gingival tissue. Quintessence Int 53-59, 2016.

8. Al Hamad KQ, Azar WZ, Alwaeli HA and Said KN: A clinical study on the effects of cordless and conventional retraction techniques on the gingival and periodontal health. J Clin Periodontol 35: 1053-1058, 2008.

9. Goldberg PV, Higginbottom FL and Wilson TG: Periodontal considerations in restorative and implant therapy. Periodontol 2000 25: 100-109, 2001

10. Nowakowska D and Raszewski Z: The $\mathrm{pH}$ value of conventional and experimental gingival margin retraction medicaments. Dent Med Probl 47: 76-80, 2010.

11. Katareva I, Georgieva K, Simeonov S, Doychinova M and Tonchev T: Application of xylometazoline for chemo-mechanical retraction of the free gingiva. J of IMAB 21: 849-852, 2015.

12. Gerhard-SzépS: Cytotoxicpotential ofHCl-xylometazoline-based gingival retraction solutions. DZZ 2ß16: 38-50, 2016.

13. Buchanan WT, Thayer KE and Yoder JL: Systemic effects of epinephrine-impregnated retraction cord in fixed partial denture prosthodontics. J Am Dent Assoc 104: 482-484, 1982.

14. Bader JD, Bonito AJ and Shugars DA: A systematic review of cardiovascular effects of epinephrine on hypertensive dental patients. Oral Surg Oral Med Oral Pathol Oral Radiol Endod 93: 647-653, 2002.

15. Fazekas A,Csempesz F, Csabai Z and Vág J: Effects of pre-soaked retraction cords on the microcirculation of the human gingival margin. Oper Dent 27: 343-348, 2002.

16. Csillag M, Nyiri G, Vag J and Fazekas A: Dose-related effects of epinephrine on human gingival blood flow and crevicular fluid production used as a soaking solution for chemo-mechanical tissue retraction. J Prosthet Dent 97: 6-11, 2007.

17. Bowles WH, Tardy SJ and Vahadi A: Evaluation of new gingival retraction agents. J Dent Res 70: 1447-1449, 1991.

18. Kopač I, Cvetko E, Pavlica Z and Marion L: Gingival tissue inflammatory response following treatment with chemical retraction agents in Beagle dogs. Pflugers Arch 442 (6 Suppl 1): R145-R146, 2001.

19. Kopač I, Cvetko E and Marion L: Gingival inflammatory response induced by chemical retraction agents in beagle dogs. Int J Prosthodont 15: 14-19, 2002.

20. Kostić I, Mihailović D, Najman S, Stojanović S and Kostić M: The rabbit gingival tissue response to retraction liquids and tetrahydrozoline. Vojnosanit Pregl 71: 46-51, 2014.

21. Kopač I, Batista U, Cvetko E and Marion L: Viability of fibroblasts in cell culture after treatment with different chemical retraction agents. J Oral Rehabil 29: 98-104, 2002.

22. Kopač I, Sterle M and Marion L: Electron microscopic analysis of the effects of chemical retraction agents on cultured rat keratinocytes. J Prosthet Dent 87: 51-56, 2002.

23. Liu CM, Huang FM, Yang LC, Chou LS, Chou MY and Chang YC: Cytotoxic effects of gingival retraction cords on human gingival fibroblasts in vitro. J Oral Rehabil 31: 368-372, 2004.

24. Liu J, Zhang XM, Hao PJ, Hui M and Yu HY: Comparison of cytotoxicity between chemical retraction agents on human gingival fibroblasts in vitro. Hua Xi Kou Qiang Yi Xue Za Zhi 27: 202-205, 2009 (In Chinese).

25. Nowakowska D, Saczko J, Kulbacka J and Choromanska A: Dynamic oxidoreductive potential of astringent retraction agents. Folia Biol (Praha) 56: 263-268, 2010.

26. Nowakowska D, Saczko J, Kulbacka J, Choromanska A and Raszewski Z: Cytotoxic potential of vasoconstrictor experimental gingival retraction agents: In vitro study on primary human gingival fibroblasts. Folia Biol (Praha) 58: 37-43, 2012.

27. Dominiak M and Saczko J: Method of primary culture of human fibroblasts for autologous augmentation.PL patent 209784B1. Filed December 4, 2006, issued October 31, 2011. 
28. Nowakowska D, Panek H, Nowakowska M and Nowakowska A: Gingival retraction-survey results of Polish dentists. Part 2. Clinical habits related to retraction procedures. Protet Stomatol 56: 361-366, 2006.

29. Nowakowska D, Saczko J, Bieżuńska-Kusiak K, Choromańska A, Dubińska-Magiera M, Ziętek M and Kulbacka J: The influence of retraction agents on cytoskeleton reorganization and oxidative stress in primary human gingival fibroblasts (HGFs). Arch Oral Biol 59: 341-348, 2014.

30. Fedchenko N and Reifenrath J: Different approaches for interpretation and reporting of immunohistochemistry analysis results in the bone tissue-a review. Diagn Pathol 9: 221, 2014.

31. Walker RA: Quantification of immunohistochemistry-issues concerning methods, utility and semiquantitative assessment I. Histopathology 49: 406-410, 2006.
32. Zabel M (ed): Immunocytochemia. 1st edition.PWN, Warszawa 1999.

33. Kim MC, Neal DM, Kamm RD and Asada HH: Dynamic modeling of cell migration and spreading behaviors on fibronectin coated planar substrates and micropatterned geometries. PLoS Comput Biol 9: e1002926, 2013.

(i) $\Theta$ This work is licensed under a Creative Commons Attribution-NonCommercial-NoDerivatives 4.0 International (CC BY-NC-ND 4.0) License. 